Check for updates

Cite this: RSC Adv., 2018, 8, 39657

Received 25th September 2018 Accepted 14th November 2018

DOI: $10.1039 / c 8 r a 07965 j$

rsc.li/rsc-advances

\title{
Underwater superoleophobic polyurethane-coated mesh with excellent stability for oil/water separation $\dagger$
}

\author{
Xianhou Yang, ${ }^{\text {ac }}$ Daning Lang, ${ }^{a}$ Ziyuan Wang, ${ }^{a}$ Jingjing Cao, ${ }^{a}$ Ronglan Wu (D) *a \\ and Wei Wang (iD *b
}

\begin{abstract}
Oil/water separation has been a challenge in chemical engineering for various applications. There are numbers of studies on using coated metal meshes as a filter for oil/water separation. However, water resistance, chemical (such as: acid, base, and fouling) resistance and heat resistance for coating materials need further exploration, especially in terms of the durability of the coating materials. In this study, we synthesized a new coating material, hydrophilic polycarbonate polyurethane (HPCPU). We used HPCPU to chemically modify a steel mesh, and the mesh exhibits superhydrophilic and underwater superoleophobic properties. The HPCPU coated mesh shows excellent capacity for oil/water separation with a separation efficiency higher than $99.99 \%$ even after 40 cycles of separation. The coating material also exhibits excellent properties of water resistance, heat resistance, and chemical resistance. Moreover, the HPCPU-coated mesh exhibits a strong durability. For example, the separation efficiency for various oil/water mixtures remains higher than $99.7 \%$ after the HPCPU-coated mesh has been soaked in water for 30 days, hot water for 5 days, oils for 5 days, $0.5 \mathrm{M} \mathrm{HCl}$ solution, $0.5 \mathrm{M} \mathrm{NaOH}$ solution and $0.5 \mathrm{M}$ $\mathrm{NaCl}$ solution for 24 hours.
\end{abstract}

\section{Introduction}

Oil/water separation has become an indispensable and pressing challenge due to the growing waste of oil/water mixtures caused by the frequent oil spills and the emission of industrial waste water. ${ }^{1}$ Effective technologies are needed to remove, recover and clean up oil spills or oil slicks from the surface of water. ${ }^{2}$ So far, a number of methods are applied in handling the water/oil separation in real oil spill accidents, such as shimming, using dispersants, in situ burning, and other manpower-intensive technologies. ${ }^{3}$ The environmental and economic concerns of treating oil spills have encouraged many researchers to search for and find eco-friendly solutions to separate oil/water mixtures, such as adsorption, bioremediation, and filtration. ${ }^{4-11}$ Among these technologies, filtration is the most frequently-used method due to certain advantages, such as high flux, non-pollution, low energy consumption, eco-friendliness and easy-operation. ${ }^{6}$

${ }^{a}$ Key Laboratory of Oil and Gas Fine Chemicals, College of Chemistry and Chemical Engineering, Xinjiang University, Urumqi, 830046, China. E-mail: wuronglan@163.com ${ }^{b}$ Department of Chemistry and Center for Pharmacy, University of Bergen, Bergen, 5020, Norway.E-mail: wei.wang@uib.no

'Yibin Tianyuan Group Co., Ltd., Yibin, 644004, China

$\dagger$ Electronic supplementary information (ESI) available: The videos for oil/water separations are available in the supporting information. See DOI: 10.1039/c8ra07965j
With regard to wettability, the coating materials used in filtration are mainly divided into two categories. One is the 'oilremoving' type with the properties of superoleophilicity and superhydrophobicity; ${ }^{12-21}$ the other is 'water-removing' type with the properties of superhydrophilicity and superoleophobicity, ${ }^{22-25}$ or the properties of superhydrophilicity and underwater superoleophobicity. ${ }^{26-32}$ In recent studies, the coating materials for the 'water-removing' type have attracted great attention due to the advantages of outstanding stain resistance and industrial applicability, in particularly, the coating materials with superhydrophilicity and underwater superoleophobicity. The superhydrophilic and underwater superoleophobic material is usually prepared, then covering the hydrophilic material on the surface of the porous material by physical coating or chemical grafting. To achieve the superhydrophilicity and underwater superoleophobicity, many materials have been used as membrane substrates of filtration, including metal meshes, fabrics, ${ }^{33-35}$ foams, ${ }^{13,36}$ nylon meshes, ${ }^{37}$ aerogel, ${ }^{38}$ sponges $^{39-41}$ and etcetera. Among them, metal meshes, such as stainless-steel mesh and copper mesh, exhibit superior mechanical properties and feasibility. Whereas, the surface of metal meshes is difficult to modify chemically to achieve the hydrophilicity. Often, the surface is modified physically to obtain the hydrophilicity by coating with hydrophilic hydrogels, ${ }^{42,43}$ graphene oxide, ${ }^{44,45}$ titania, ${ }^{46}$ zinc oxide, ${ }^{47}$ palygorskite, ${ }^{30}$ chitosan $^{48}$ and etc. Due to the superhydrophilic property and lacking the chemical bonds between the metals and the coating materials, the coating easily 
falls off from the mesh after being in the water for long time. Consequently, the water-resisting property of such meshes is very poor. For copper meshes, the surface could be chemically modified to yield the hydrophilicity. The hydrophilic compounds, generated on the surface of the copper mesh by chemical reactions, such as: copper hydroxide, copper oxide, etc. ${ }^{49-52}$ are easy to react with acids, leading to the damage of the copper mesh, implying a deficiency of acid-resistance. The practical application calls for new coating materials with the new properties, such as remarkable chemical stability and waterresistance, in addition to high flux, low environment pollution, low energy consumption, and simple operation. There are extremely few relevant reports on promising metal meshes with superhydrophilic and underwater superoleophobic properties.

In the previous studies in our group, we found that the polycarbonate polyurethane (PCPU) exhibits excellent water resistance, heat resistance, and weatherability. ${ }^{53-56}$ Here, we prepared two layers of coating coated mesh with superhydrophilicity and underwater superoleophobicity. First, we synthesized inner layer that PCPU with terminal group of $\mathrm{C}=\mathrm{C}$ and then coated it onto the stainless steel mesh (PCPU-coated mesh). Next, outer layer, poly-hydroxyethyl acrylate (PHEA), was grafted on PCPU-coated mesh by free radical polymerization to obtained hydrophilic PCPU-coated (HPCPU-coated) mesh. Under the joint effect of strong adhesion between water resistant, weatherable inner-layer and stainless steel mesh and chemical crosslinking between outer layer and inner layer, the coating material exhibits strong water resistance, heat resistance and acid/base-resistance in addition to the high separation efficiency (>99.7\%) for various oil/ water mixtures. The high separation efficiency was maintained after the coated meshes being treated in water for 30 days, in hot water for 5 days, in $0.5 \mathrm{M} \mathrm{HCl}, 0.5 \mathrm{M} \mathrm{NaOH}$, and $0.5 \mathrm{M} \mathrm{NaCl}$ solutions for 24 hours, and in various oils for 5 days. To our knowledge, this is the first time that the metal meshes for oil/ water separation exhibits such properties. In addition, the synthetic route for the coating material is very simple, depicting a promising application in large-scale production.

\section{Materials and methods}

\subsection{Materials}

Polycarbonate diol was purchased from Jinjiuchen Chemicals (Tianjin, China) with the average molecular weight of 3000 . Ammonium persulfate (APS) was purchased from Hongyan Chemicals (Tianjing, China). Absolute ethanol and $\mathrm{N}, \mathrm{N}$-dimethylformamide (DMF) were purchased from Oubokai Co. Ltd. (Tianjing, China). Isophorone diisocyanate (IPDI), 1,4-butanediol (BDO), 2-hydroxyethyl acrylate (HEA), dibutyltin dilaurate, sodium hydroxide and hydrochloric acid were purchased from Aladdin Chemicals (Shanghai, China). All chemicals were used as received. Milli-Q water with resistivity of $18.2 \mathrm{M} \Omega \mathrm{cm}$ was used for all experiments.

\subsection{Synthesis of polycarbonate polyurethane (PCPU)}

Scheme 1 shows the synthetic route of PCPU. Polycarbonate diol (PCDL, $1.8 \mathrm{~g}$ ) and isophorone diisocyanate (IPDI, $4.45 \mathrm{~g}$ ) were mixed in a flask, and reacted at $70{ }^{\circ} \mathrm{C}$ for 2 hours. The solution was then cooled down to $60{ }^{\circ} \mathrm{C}$, and 1,4-butanediol $(1.13 \mathrm{~g})$ and DMF $(10 \mathrm{~mL})$ were added into the solution. The mixture was stirred at $60{ }^{\circ} \mathrm{C}$ for 2 hours, and yielded PCPU with the end group of isocyanate group (PCPU-NCO). Then, 2-hydroxyethyl acrylate (HEA, $1.60 \mathrm{~g}$ ) and dibutyltin dilaurate $(0.04 \mathrm{~g})$ were added into the mixture. The mixture was stirred at $60{ }^{\circ} \mathrm{C}$ for another hour to obtain PCPU with carbon-carbon double bonds at the end of the polymer chain (PCPU-C $=\mathrm{C}$ ).

\subsection{Preparation hydrophilic polycarbonate polyurethane (HPCPU) coated mesh}

Scheme 2 shows the preparation of HPCPU-coated mesh. A steel-mesh $\left(5 \times 5 \mathrm{~cm}^{2}, 300\right.$ mesh) was first cleaned with water and ethanol thoroughly, and dried. Then, the PCPU $(2 \mathrm{~mL})$ and DMF $(6 \mathrm{~mL})$ were mixed in a beaker. The steel-mesh was immersed into the solution for 10 seconds. The PCPU-coated mesh was obtained by drying the mesh at $70{ }^{\circ} \mathrm{C}$ for 30 minutes. Afterwards, the PCPU-coated mesh was immersed in an aqueous solution of APS $\left(0.5 \mathrm{mg} \mathrm{mL}{ }^{-1}, 40 \mathrm{~mL}\right)$ at $70{ }^{\circ} \mathrm{C}$ for 10 minutes. Then, HEA (1.5 g) was added into the solution, and the mesh was kept in the solution for 6 hours at $70{ }^{\circ} \mathrm{C}$ to obtain HPCPU-coated mesh.

\subsection{Characterization}

The chemical compositions of PCPU-CNO and PCPU-C $=\mathrm{C}$ were examined by Fourier transform infrared spectroscopy (FTIR, Prestige-21, Shimadzu, Japan). The HPCPU-coated mesh was investigated by thermal gravimetric analyzer (TG-STA7300, Hitachi, Japan). The morphology of the steel-mesh, PCPUcoated mesh, and HPCPU-coated mesh were characterized by scanning electron microscopy (SEM-TM3030, Hitachi, Japan). Underwater oleophobicity of the PCPU-coated mesh and the HPCPU-coated mesh was characterized by contact angle measurements (JJ2000B, Zhongchen, Shanghai, China) at room temperature. The measurements were carried out at minimum five positions.

\section{Results}

\subsection{FT-IR characterization of PCPU-CNO and PCPU-C $=\mathrm{C}$}

In the synthesis, the introduction of $\mathrm{C}=\mathrm{C}$ to $\mathrm{PU}$ is essential to achieve a stable and hydrophilic coating since the $\mathrm{C}=\mathrm{C}$ bonds can be easily crosslinked with HEA by free-radical polymerization. This process was characterized by FT-IR. The FT-IR spectra of PCPU-CNO and PCPU-C $=\mathrm{C}$ are shown in Fig. 1a. For the PCPU-NCO and PCPU- $=\mathrm{C}$, the characteristic adsorptions are identified as follows: stretching vibrational absorption of secondary amino groups (-NH) of carbamate groups (-HNCOO-) at $3327 \mathrm{~cm}^{-1}$; methylene $\left(-\mathrm{CH}_{2}-\right)$ at $2950 \mathrm{~cm}^{-1}$; carbonyl groups $(-\mathrm{C}=\mathrm{O})$ of carbamate groups (-HNCOO-) at $1718 \mathrm{~cm}^{-1}$; ether groups (-C-O-) of carbamate groups (-HNCOO-) at $1250 \mathrm{~cm}^{-1}$; deformed vibrational absorption peak of secondary amino groups (-NH) of carbamate groups (-HNCOO-) at $1535 \mathrm{~cm}^{-1}$. In addition, for the PCPU-CNO, a sharp -NCO absorption peak is present at $2267 \mathrm{~cm}^{-1}$, and after the last step in the synthesis, the 
<smiles>CC(C)C1(CN=C=O)CC(N=C=O)CC(C)(C)C1</smiles><smiles>O=POOC(=O)OPO</smiles>

PCDL

$70^{\circ} \mathrm{C}, 2 \mathrm{~h}$
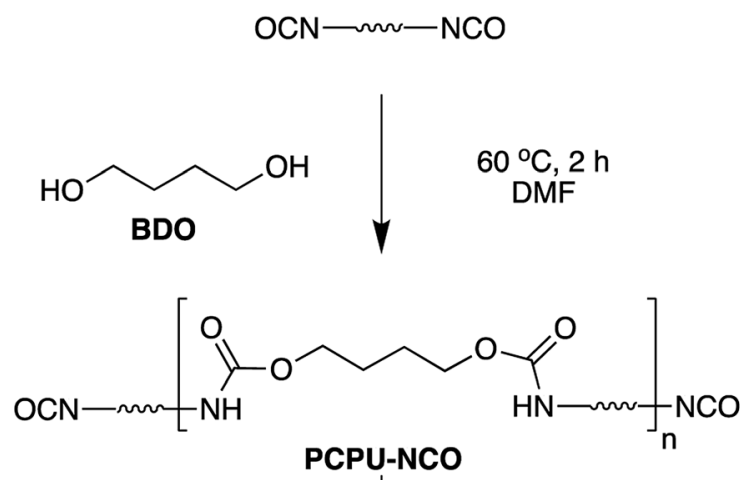<smiles>C=CC(=O)OCCO</smiles>

$60^{\circ} \mathrm{C}, 1 \mathrm{~h}$

Dibutyltin dilaurate

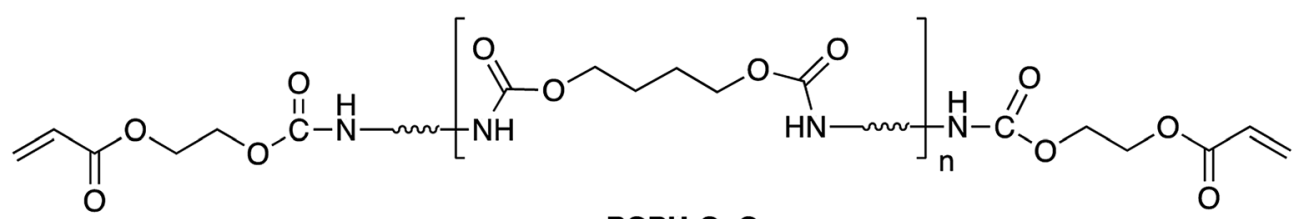

PCPU-C $=\mathrm{C}$

Scheme 1 The reaction route for the synthesis of PCPU.

absorption peak at $2267 \mathrm{~cm}^{-1}$ was completely disappeared for the $\mathrm{PCPU}-\mathrm{C}=\mathrm{C}$, indicating that the HEA successfully reacted with PCPC-CNO, which further resulted a HPCPU-coated mesh with superhydrophilicity and underwater superoleophobicity.

\subsection{Thermogravimetric analysis of the HPCPU coated mesh}

Because the coating will affect the flux as well as the preparation cost, HPCPU on the coated mesh was investigated by thermogravimetric analysis. As shown in Fig. 1b, the weight of the coated mesh started decreasing at $175{ }^{\circ} \mathrm{C}$. When temperature reached $390{ }^{\circ} \mathrm{C}$, the weight of coated mesh decreased to $97.4 \%$, and leveled out, suggesting that the quality of coating accounts for $2.6 \%$ of the quality of the coated mesh.

\subsection{Mesh morphology}

Fig. 2 shows the SEM images of the untreated steel-mesh, the PCPU-coated mesh, and the HPCPU-coated mesh. The untreated mesh shows a smooth surface of steel material (Fig. 2a), and the size of mesh is around $50 \mu \mathrm{m}$ (Fig. 2b). The untreated mesh was simply dipped in the solution of PCPU for ten seconds, and left for drying. Due to the high viscosity of the solution, a fine layer of PCPU was covered on the steel mesh and formed a uniform film (Fig. 2c). The enlarged image of the film shows a smooth feature of the PCPU coated on the mesh (Fig. 2d). Small holes on the scale of $30 \mu \mathrm{m}$ can be found on the mesh. The coating in the first step is a physical one in nature. The coated layer is dependent on the viscosity of the PCPU, and

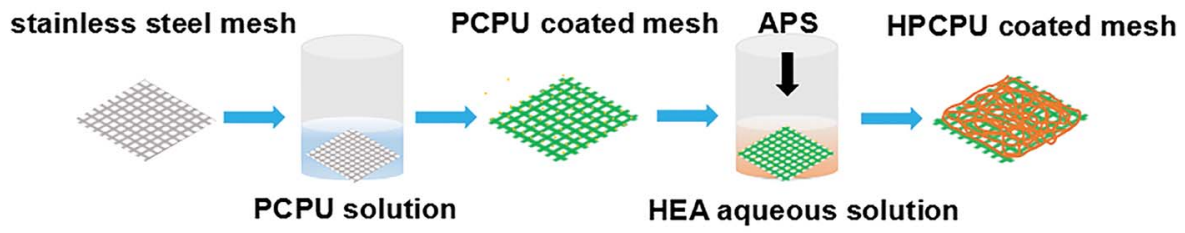

Scheme 2 The preparation of HPCPU-coated mesh. 

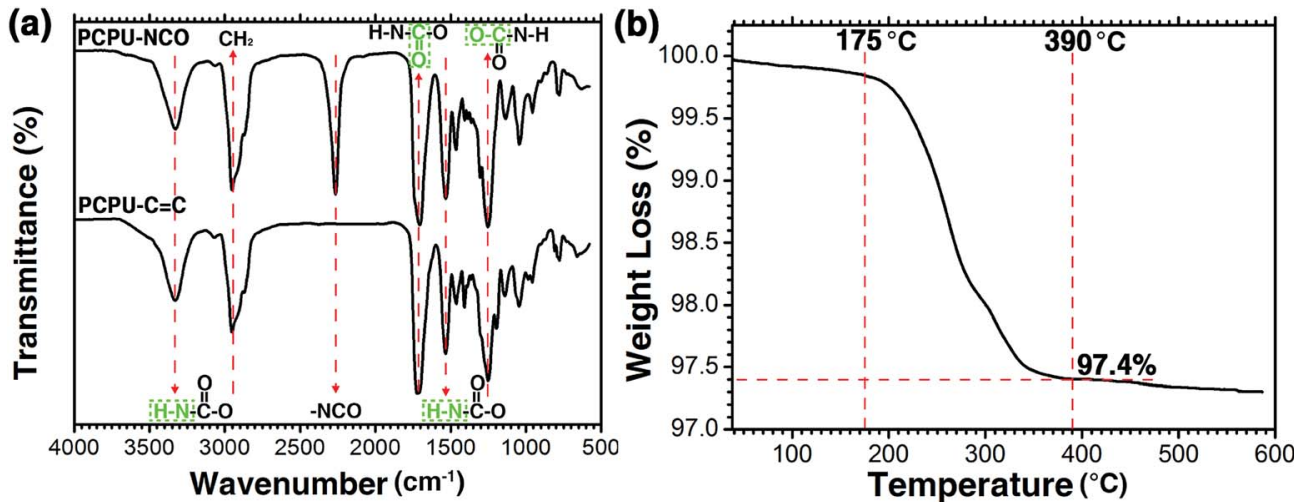

Fig. 1 (a) The FT-IR spectra of PCPU-CNO and PCPU-C=C. (b) Thermogravimetric analysis of the HPCPU-coated mesh.

the coated layer may not be stable. In the synthesis, the reactive double bonds were introduced in the last step (Scheme 1). The acrylate group in PCPU can be easily crosslinked with HEA by free-radical polymerization to obtain two layer of coating HPCPU-coated mesh. Besides obtaining the hydrophilicity, the chemically crosslinked film is much more robust as it shows in


Fig. 2 SEM images of the as-prepared mesh: (a) the untreated steelmesh; (b) a pore of the untreated stain-steel mesh; (c) the PCPUcoated mesh; (d) the enlarged view of the surface of PCPU on the coated mesh; (e) the HPCPU-coated mesh; ( $f$ ) the enlarged view of the surface of HPCPU on the coated mesh. the later tests. The surface of the PCPU-coated mesh is also undergone a noticeable change (Fig. 2d). Comparing to the PCPU-coated mesh, the HPCPU-coated mesh shows evenly distributed pores with the size of $50 \mu \mathrm{m}$ (Fig. 2e). The enlarged view of the HPCPU surface displays a rough feature (Fig. 2f). Micrometer-scaled groove patterns formed on the surface of the HPCPU-coated mesh.

\subsection{The wettability of the PCPU and HPCPU-coated meshes}

Chemical composition and the micro/nano-structure of a solid surface are the two factors that affect the wettability, and the wettability will further affect the separation efficiency of oil/ water mixture. Thus, the wettability of the PCPU and HPCPUcoated meshes were characterized by the contact angle measurements (Fig. 3). In air, a droplet of water placed on the surface of the PCPU-coated mesh (Fig. 3a). The contact angle is about $60^{\circ}$. Whereas, the contact angle of water on the HPCPUcoated mesh is $0^{\circ}$ (Fig. $3 \mathrm{~b}$ ). Under water, a droplet of dichloromethane placed on the surface of the PCPU-coated mesh (Fig. 3c). The contact angle is about $45^{\circ}$, indicating the PCPU-coated mesh can be wetted by oil (dichloromethane); whereas, the HPCPU-coated mesh shows an underwater superoleophobic feature because the contact angle of water/ dichloromethane on the HPCPU-coated surface is larger than $150^{\circ}$ (Fig. 3d). These results indicate that the PCPU-coated mesh was successfully modified and the surface of the mesh was the superhydrophilic and underwater superoleophobic.

We further measured the contact angles of light oils/water beneath the surface of the HPCPU-coated mesh (Fig. 3e). In all the measurements, the contact angles are in the range of $154^{\circ}$ to $162.5^{\circ}$, indicating the underwater superoleophobic feature of the HPCPU-coated mesh. This underwater superoleophobic feature can be attribute to the cooperative effect of the hydrophilicity of the massive hydroxyl groups on the grated poly-HEA and the rough microstructure of the coating (Fig. 2f). Due to this cooperative effect, water can be trapped in the rough microstructure and forms a hydrophilic layer, which provides a strong repulsive force to oil due to the difference of the surface tension. As a consequence, the coating material can also provide a barrier from oil stains, thus, prolonging its service life. 

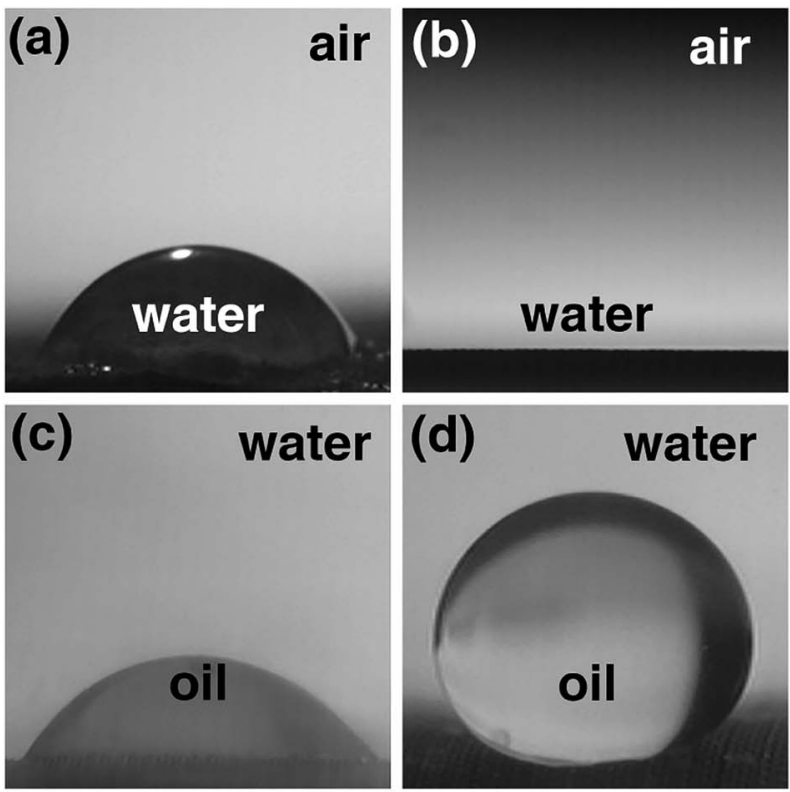

(e)

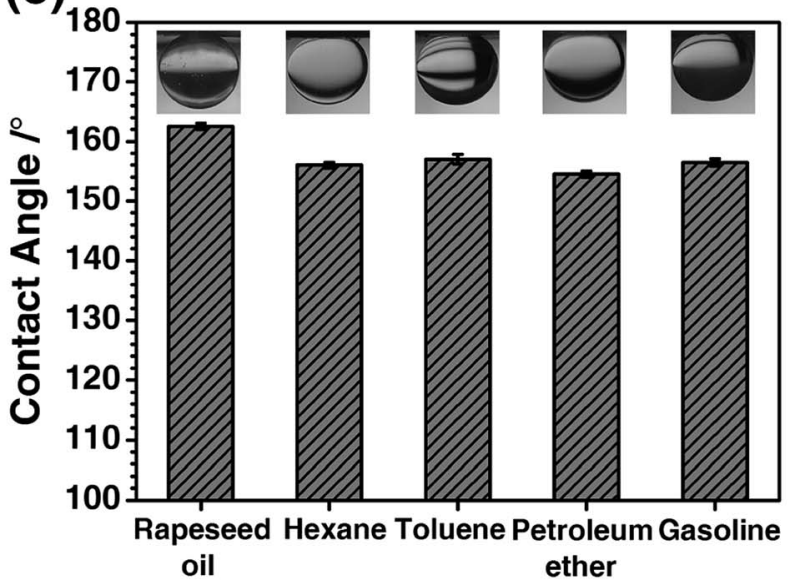

Fig. 3 The wettability of ( $a$ and $c$ ) the PCPU-coated mesh and (b, $d$ and e) the HPCPU-coated mesh: (a) the contact angle of water on the PCPU-coated mesh in air; (b) the contact angle of water on the HPCPU-coated mesh in air; (c) the contact angle of dichloromethane on the PCPU-coated mesh under water (d) the contact angle of dichloromethane on the HPCPU-coated mesh under water; (e) the contact angles of light oils beneath the surface of the HPCPU-coated mesh under water.

Subsequently, the adhesion of underwater oil on the surface of the HPCPU-coated mesh was also studied. The process of adhesion of dichloromethane oil on HPCPU-coated mesh was recorded (Fig. 4). In the first two steps, the needle was slowly moved towards the HPCPU-coated mesh. In the second picture, the dichloromethane contact with the HPCPU-mesh. When we retrieved the needle, the liquid drop was easily detached from the surface of the mesh. Based on this results, we may conclude the HPCPU-coated mesh has excellent resistance from oil contamination.

\subsection{Oil/water separation}

To test the capability of oil/water separation for the coated meshes, we carried out a series of proof-of-concept studies. The apparatus is shown in Fig. 5. The pre-wetted mesh was fixed between two volumetric cylinders. Mixture of $n$-hexane and water $(1: 1 \mathrm{v} / \mathrm{v})$ was poured in the cylinder. Fig. $5 \mathrm{a}-\mathrm{c}$ show the process of oil/water separation for the PCPU-coated mesh. Both water and hexane quickly permeated through the mesh and dropped into a beaker (Video S1 in the ESI $\dagger$ ). However, the oil/ water separation by using the HPCPU-coated mesh exhibited a completely different scenario (Fig. $5 \mathrm{~d}-\mathrm{f}$, and Video S2 in the ESI $\dagger$ ). In Fig. 5f, water permeated through the mesh, whereas hexane retained above the mesh due to the superhydrophilic and underwater superoleophobic features of the coated mesh. Based on the visual inspection, no oil drop was found in the beaker, indicating high separation efficiency.

The reuse of the HPCPU-coated mesh (Fig. 6a) and a variety of oils (Fig. 6b) were tested for the oil/water separation by using the HPCPU-coated mesh. The separation efficiency $(\eta)$ can be quantitatively calculated as follows: $\eta=\left(1-\frac{m_{2}}{m_{1}}\right) \times 100 \%$, where $m_{1}$ and $m_{2}$ are the mass of oil in water before and after the separation, respectively. The separation efficiency for the hexane/water system was $99.994 \%$ on the first separation, and maintained on the very high level after even 40 separation cycles (Fig. 6a). We further evaluated the durability of the mesh on the oil/water separation. After 500 cycles of oil/water separation, the separation efficiency retained above $99.7 \%$ the separation efficiency. Fig. $6 \mathrm{~b}$ shows the separation efficiency for the oil/water mixtures of other five types of oil. The separation efficiencies are all higher than $99.7 \%$.

The intrusion pressure of the oils on the HPCPU-coated mesh is a parameter that assesses the separation ability of coated meshes. Based on the definition: $P=\rho g h_{\max }$, the experimental intrusion pressure of the oils on the HPCPUcoated mesh was calculated, where $\rho$ is the density of the oil, $g$ is the acceleration of gravity and $h_{\max }$ is the maximum height of the oil that the coated mesh can support. The average maximum heights of 5 measurements for a series of oil are obtained, and all of them are higher than $8 \mathrm{~cm}$ (Fig. 6c). Correspondingly, the experimental intrusion pressure is calculated to be over $0.7 \mathrm{kPa}$ (Fig. 6d).

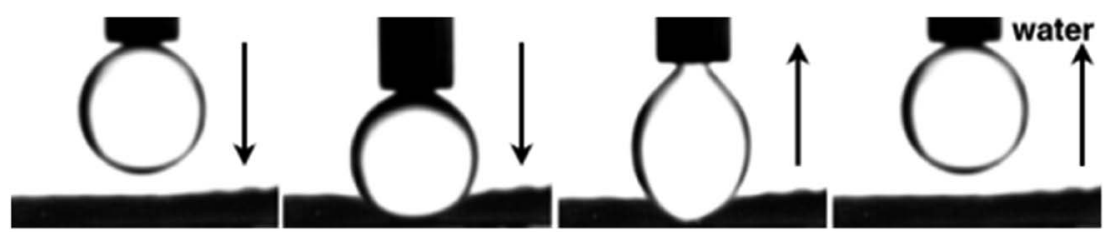

Fig. 4 The adhesion test of underwater dichloromethane on the surface of the HPCPU-coated mesh. 


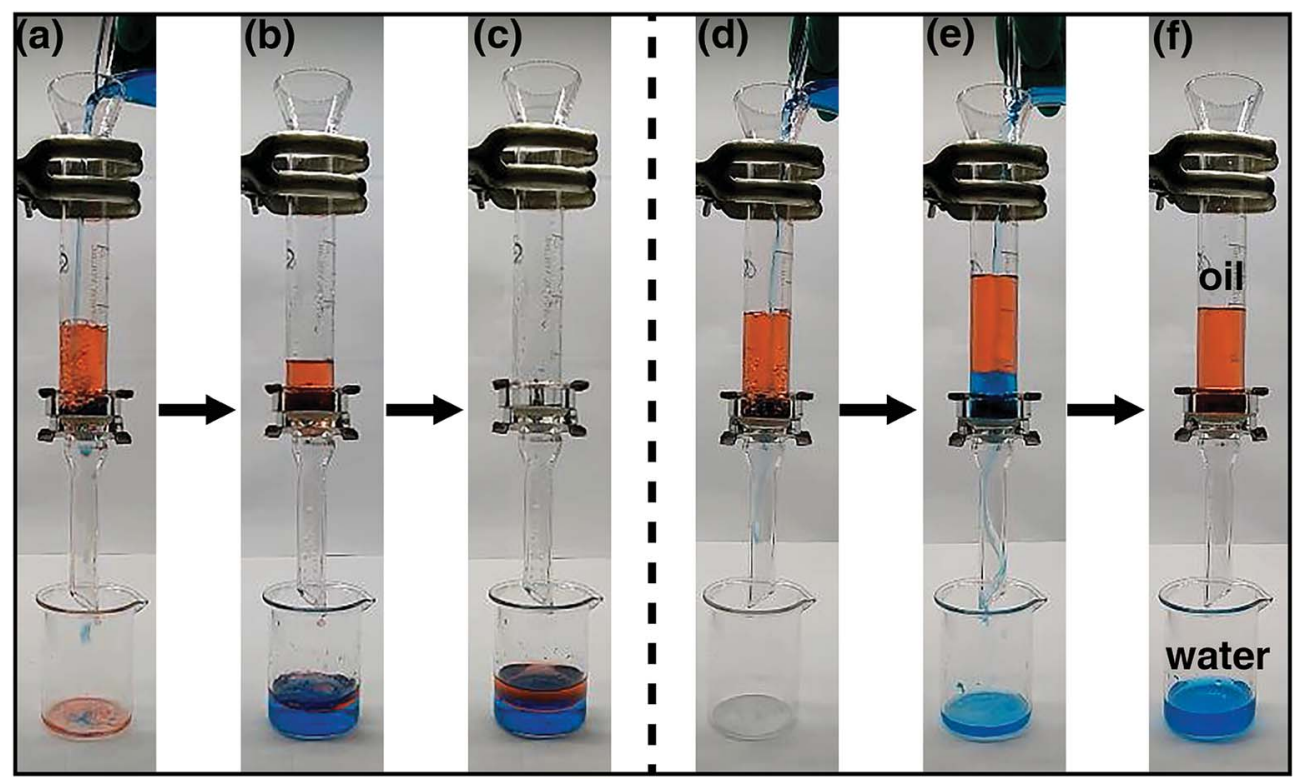

Fig. 5 Examples of the PCPU-coated and HPCPU-coated meshes for oil/water separation. The mesh is placed in between two volumetric cylinders, and fixed by a clip. Water is colored by methylene blue, and oil is colored by oil red $\mathrm{O}$. The PCPU-coated mesh was used in water/oil separation shown in (a) the beginning, (b) in the middle of process, and (c) after separation. The HPCPU-coated mesh was used for (d) the beginning, (e) in the process, and (f) after separation.

In addition to the separation efficiency and the intrusion pressure, the flux per unit time is also important. The flux of liquid $(F)$ is investigated as follows: $F=\frac{V / \pi r^{2}}{t}$ where $V$ is the volume of liquid permeated through the mesh, $r$ is the radius of the cylinder, and $t$ is the time taking for the permeation of $V \mathrm{~mL}$ liquid. In the measurements, $0.5 \mathrm{~L}$ water was used to evaluate the flux, and the average value of flux is around $25 \mathrm{~L} \mathrm{~m}^{-2} \mathrm{~s}^{-1}$.

The purity of the collected oils (rapeseed oil, hexane, toluene, petroleum ether and gasoline) was investigated by FTIR (a)

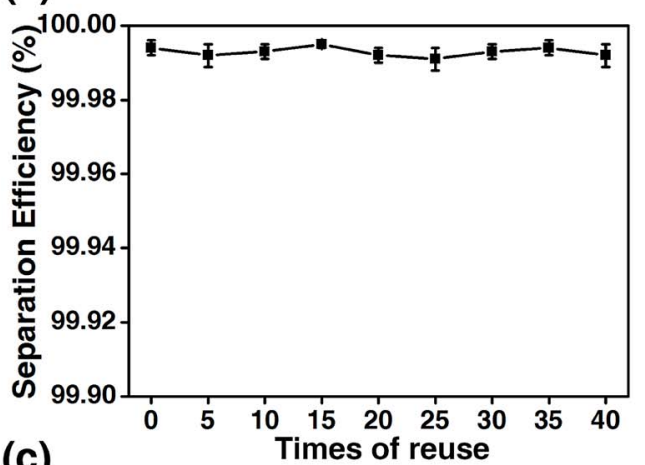

(c)

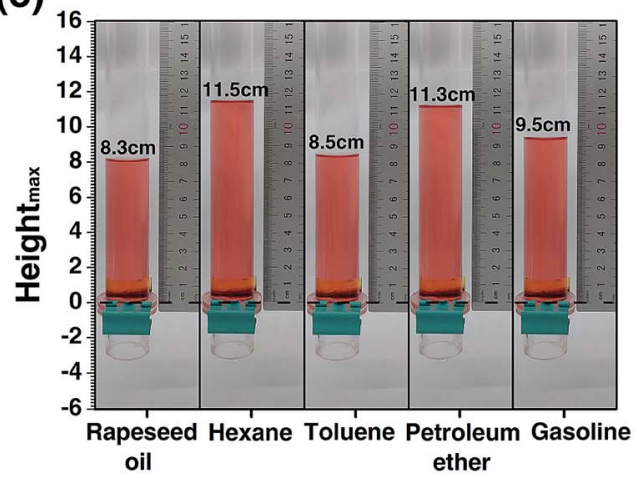

(b)

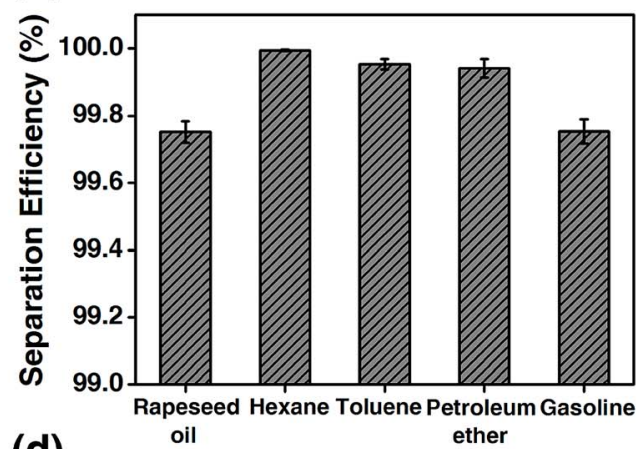

(d)

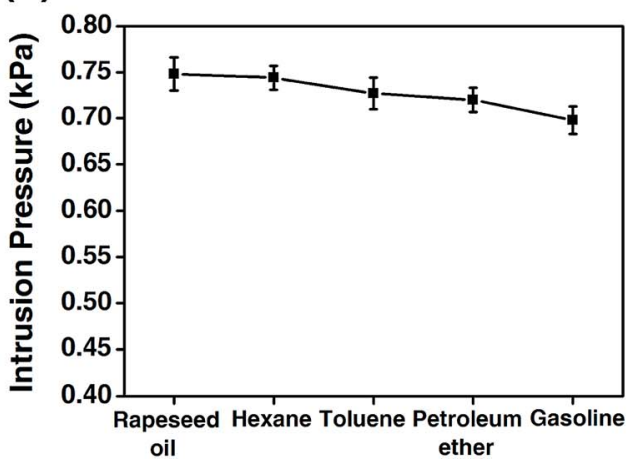

Fig. 6 The separation efficiency of the HPCPU-coated mesh: (a) the separation efficiency of hexane/water mixture for 40 cycles; (b) the separation efficiency of five types of oil/water mixtures. (c) The maximum height of the oils that the HPCPU-coated mesh can support. (d) The experimental intrusion pressure of the oils on the HPCPU-coated mesh. 

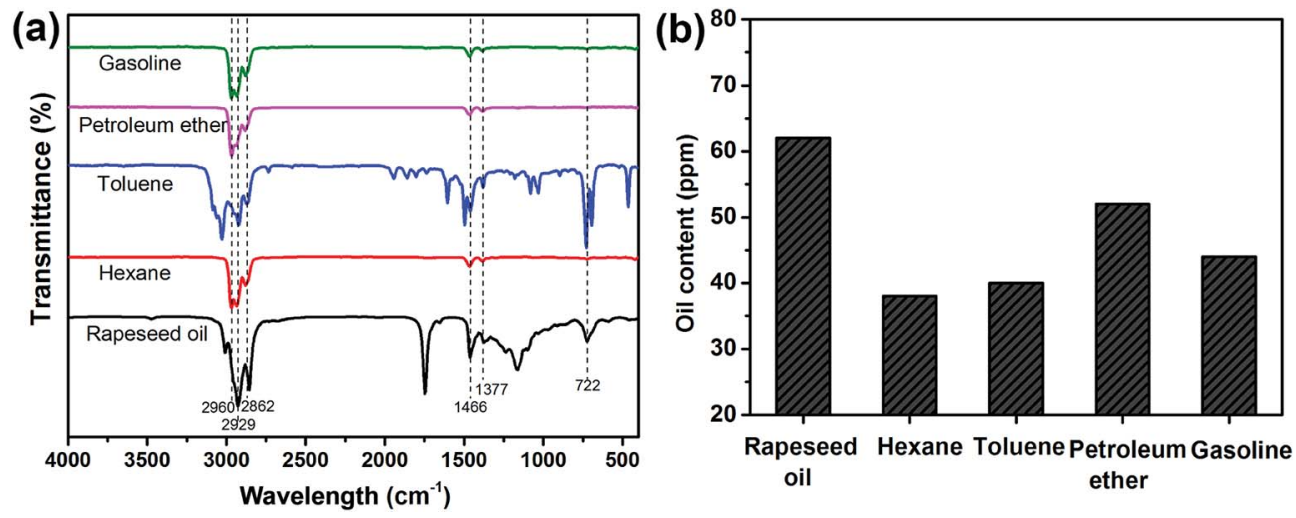

Fig. 7 (a) FTIR spectra of the separated oils. (b) Oil content in the collected water.

(Fig. 7a). All the absorption bands were owing to the characteristic vibrations of the chemical constituents of the collected oils. The bands from 2862 to $2960 \mathrm{~cm}^{-1}$ were assigned to the $-\mathrm{C}-\mathrm{H}$ asymmetric and symmetric stretching vibration of $-\mathrm{CH}_{3}$ and $-\mathrm{CH}_{2}-$ groups. The $-\mathrm{C}-\mathrm{H}$ scissoring vibration of $-\mathrm{CH}_{2}-$ groups and the $-\mathrm{C}-\mathrm{H}$ asymmetric stretching vibration of $-\mathrm{CH}_{3}$ groups were found at $1466 \mathrm{~cm}^{-1}$. The bands at $1377 \mathrm{~cm}^{-1}$ corresponded to the $-\mathrm{C}-\mathrm{H}$ symmetric bending vibration of $-\mathrm{CH}_{3}$ groups. The bands around $722 \mathrm{~cm}^{-1}$ originated from the $-\left(\mathrm{CH}_{2}\right)_{n}$ - rocking vibration and $-\mathrm{HC}=\mathrm{CH}-$ bending vibration. No trace of water was observed based on FTIR spectra, implying the high purity of the separated oils. In addition, the amount of oil in the collected water was evaluated (see Fig. 7b). According to the results, the oil content was lower than $65 \mathrm{ppm}$ for the four oil/water mixtures. Therefore, the HPCPU-coated mesh is highly efficient for oil/water separation.

\subsection{The effect of synthetic conditions on oil/water separation}

As aforementioned, chemical composition and the micro/nanostructure of the mesh affect the oil/water separation. These two factors change with the reaction conditions. Therefore, we investigated the effects of three reaction conditions on the separation efficiency, flux and intrusion pressure, which are the concentration of PCPU in DMF, the amount of HEA added as the reactant, and the reaction time.

The viscosity of PCPU is dependent on the concentration. With less PCPU in DMF, the solution becomes less viscous. In this case, when dip the mesh in the PCPU solution, there will be less PCPU coated on the mesh, and further affects the oil/water separation. In the experiment, we fix the amount of PCPU $(2 \mathrm{~mL})$ added into DMF, and increase the amount of DMF from 2 to 8 $\mathrm{mL}$, corresponding to the concentration of PCPU in the region of 8.8 to $22.8 \mathrm{wt} \%$. The amount of HEA $(1 \mathrm{~g})$ and reaction time $(10 \mathrm{~h})$ were kept constant. We observed that the flux substantially increased with the decrease of the concentration of PCPU (Fig. 8a). However, the separation efficiency remained unchanged when the concentration of PCPU was above $11 \mathrm{wt} \%$. The decrease of separation efficiency may suggest the coating on the mesh is not enough, and therefore jeopardize the oil/ water separation. Another interesting observation in Fig. 8a is that the separation efficiency shows a good correlation to the intrusion pressure. This argument will be further strengthened based on the results in Fig. $8 \mathrm{~b}$ and c.

The addition of HEA will finally crosslink the PCPU chains in the coating, which contributes to the stability of the coating as well as the separation efficiency. The PCPU concentration was kept as $11.1 \%$ and the reaction time as 10 hours, and changed

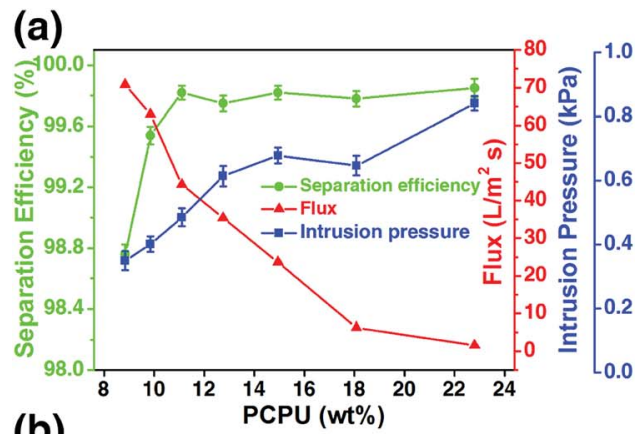

(b)

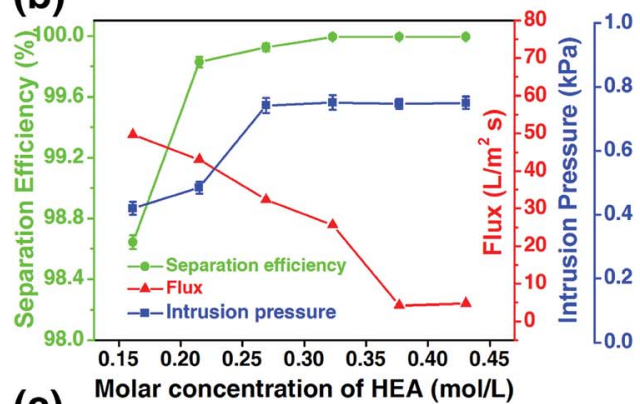

(c)

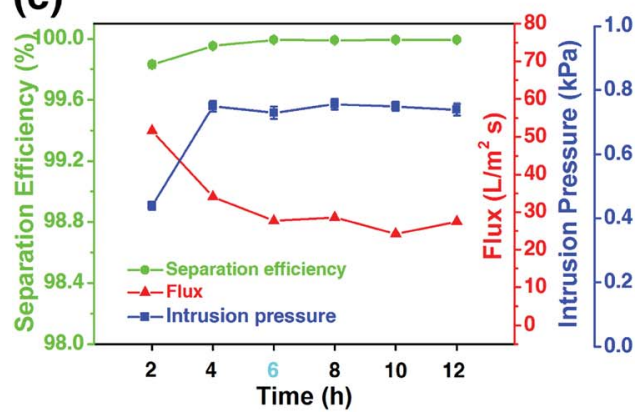

Fig. 8 The effects of (a) PCPU concentration, (b) HEA concentration, and $(c)$ reaction time on flux, separation efficiency, and intrusion pressure. 
the amount of HEA in the reaction from 0.75 to $2 \mathrm{~g}$ (corresponding to 0.16 to $\left.0.43 \mathrm{~mol} \mathrm{~L}^{-1}\right)$. A monotonic decrease of flux was observed with increasing HEA concentration in the reaction. While, the separation efficiency and intrusion pressure are both increased when HEA concentration is below $0.27 \mathrm{~mol} \mathrm{~L}^{-1}$, and afterwards levels off.

Based on these two results (Fig. 8a and b), we studied the effect of the reaction time on the oil/water separation. The results (Fig. 8c) show that reaction time has very little effect on the oil/water separation providing the enough reaction time $(>2$ hours) is given. This result is reasonable for the reaction rate of free-radical polymerization is generally large.

\subsection{Stability of the HPCPU-coated mesh in various environments}

The stability of the coated materials was investigated in harsh environments. The meshes were pretreated with $0.5 \mathrm{M} \mathrm{HCl}$ solution, $0.5 \mathrm{M} \mathrm{NaOH}$ solution, and $0.5 \mathrm{M} \mathrm{NaCl}$ solution for 24 hours. Afterwards, the meshes were used to separate the hexane/water mixture. The results are presented in Fig. 9. After the treatments, the underwater contact angle was first measured for the meshes, and Fig. 9a shows the contact angle of five types of oils on the mesh after being pretreatment. The contact angles remained the same for all types of oils, and little difference was observed for the meshes being pretreated in acidic, basic or saline solutions. The microstructure of mesh was examined by SEM. Based on the contact angle and SEM image (Fig. 9d), we conclude that the microstructure of the coated mesh is very stable in acidic, basic, and saline solutions. The separation of hexane/water mixture was performed with the meshes immersed in the solutions, respectively. Water passes through the mesh, whereas, hexane was stopped by the mesh and retained on the upper tube. The separation efficiency of the mesh was up to $99.7 \%$ for the three meshes treated with the acidic, basic, and salt solutions (Fig. 9g). The intrusion pressure (a)
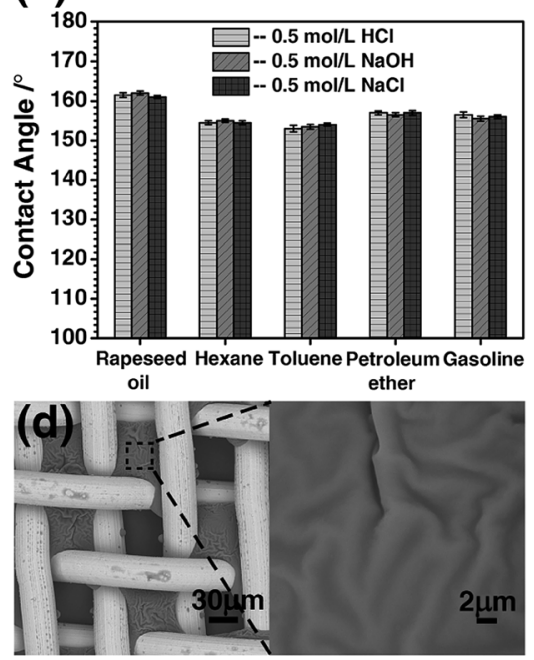

(g)

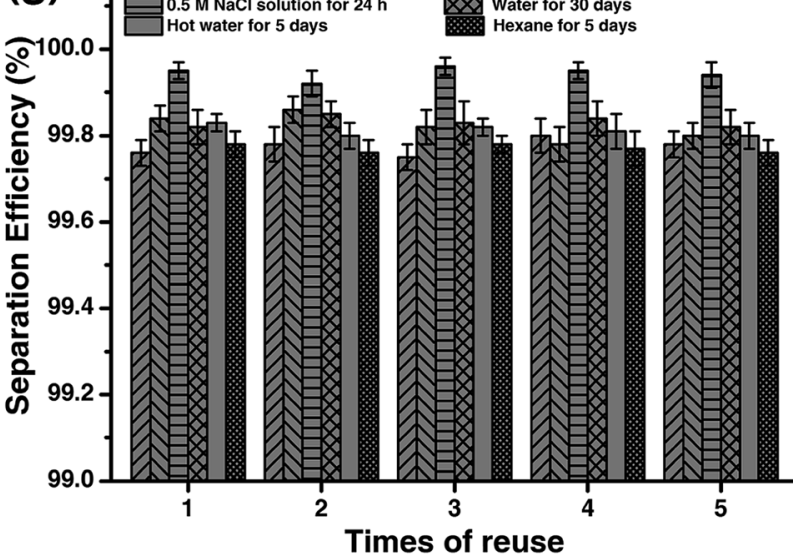

(b)

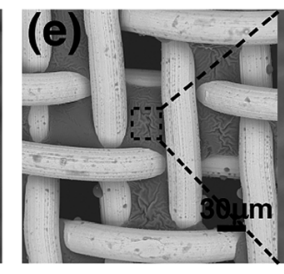

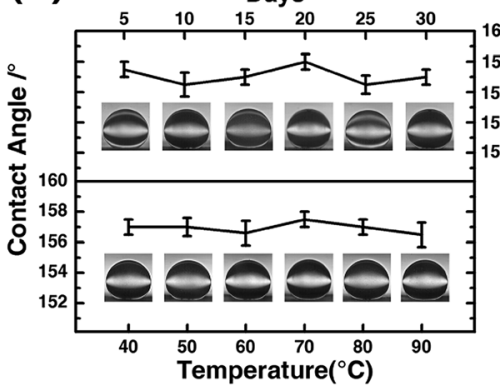

\section{(h)}

(h)

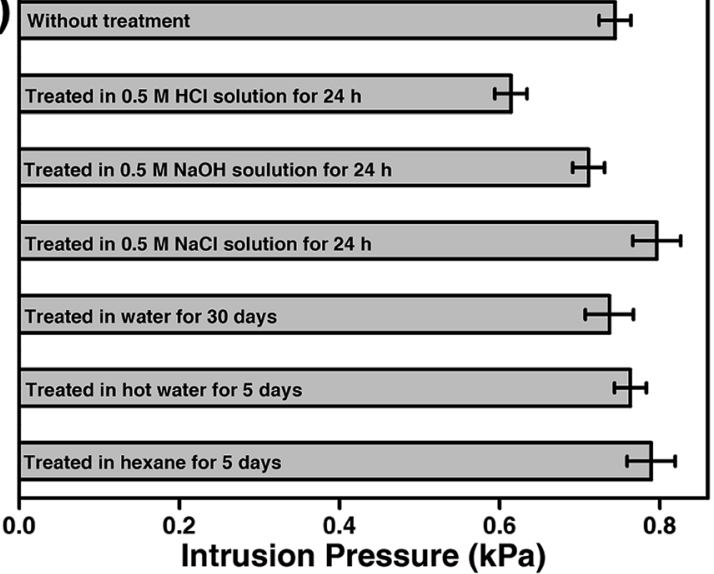

Fig. 9 (a) The underwater contact angle of five types of oils on the meshes pretreated by $0.5 \mathrm{M} \mathrm{HCl}, 0.5 \mathrm{M} \mathrm{NaOH}$, and $0.5 \mathrm{M} \mathrm{NaCl}$ solution for 24 hours, respectively; (b) the underwater contact angles of dichloromethane on the mesh pretreated in water (top). The contact angel was measured every 5 days. The underwater contact angle of dichloromethane was also measured on the mesh pretreated in hot water (bottom) (c) the underwater contact angles of dichloromethane on the meshes after pretreatment in a series of oils for 5 days. (d), (e) and ( $f$ ) are the SEM images of the mesh pretreated in $0.5 \mathrm{M} \mathrm{HCl}$ for $24 \mathrm{~h}$, hot water for 5 days and hexane for 5 days, respectively. (g) The separation efficiency of hexane/water mixture by the mesh that was pretreated in various conditions. (h) The experimental intrusion pressures of hexane passing after the mesh being pretreated in various conditions. 


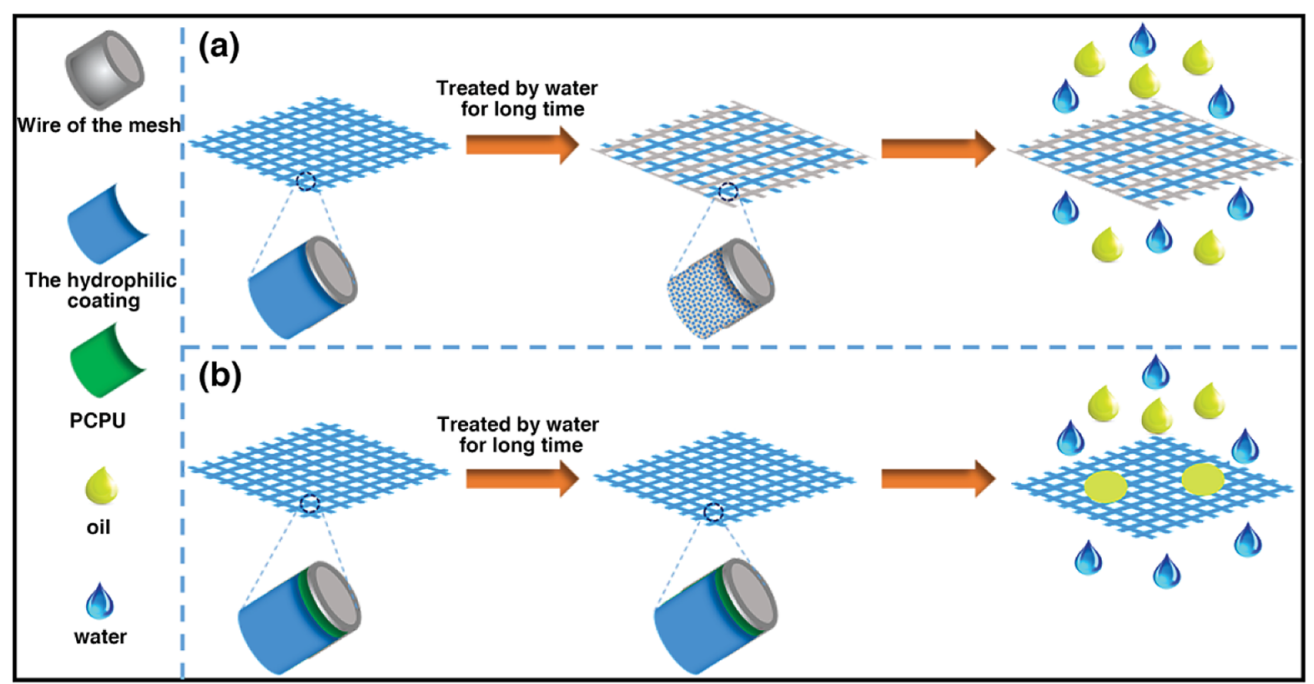

Scheme 3 A demonstration of traditional materials with underwater superoleophobicity and materials we prepared in water for long term application.

after the treatments is also maintained on the same level as it was for the original mesh (Fig. 9h).

Considering that the materials used to separate oil/water should sustain in mal conditions for practical applications, we investigated the durability of the HPCPU-coated mesh. After the mesh being immersed in water for 30 days successively, the separation of hexane/water mixture was studied. The underwater contact angle of dichloromethane remained nearly the same during the 30 days (Fig. 9b). In addition, we tested the endurance of the mesh in hot water at different temperatures for 5 days. The contact angle after the hot-water treatment is presented in Fig. 9b. We conclude that the mesh is also durable in hot water. The microstructure of the mesh also remained the same as original (Fig. 9e). We tested the separation efficiency and intrusion pressure after these two treatments. The reuse of the mesh was tested for 5 times, and the separation efficiency is higher than 99.8\% (Fig. 9g). The intrusion pressure also maintained on the same level as original (Fig. 9h).

Many of materials with underwater superoleophobicity were obtained by coating hydrophilic materials on a mesh. However, as shown in Scheme 3a, due to the superhydrophilic property of the coating and lacking covalent bonds between the matrix and the coating materials, the coating easily falls off from the mesh after being in water for long time so that the separation ability of the mesh is damaged. Here, chemically processed PCPU-C $=$ $\mathrm{C}$ with the properties of water resistant and heat-resistant as inner layer was first coated on the stainless steel mesh, and then PHEA as outer layer was grafted on the PCPU-coated mesh by free-radical polymerization. As shown in Scheme $3 \mathrm{~b}$, because the inner layer (PCPU) is water resistant, heat-resistant and adheres strongly to the stainless steel mesh, in addition, the outer layer (PHEA) is chemically crosslinked with the inner layer, the as-prepared two layer of coating shows excellent longterm stability in water, and even hot water.

Moreover, we tested the fouling resistance of the HPCPUcoated meshes. The mesh used in the experiment was treated in different oils (rapeseed oil, hexane, gasoline, toluene, and petroleum ether) for 5 days. The underwater contact angle of dichloromethane remains higher than $150^{\circ}$ (Fig. 9c). The SEM image shows that the microstructure of the mesh remains the same (Fig. 9f). The separation efficiency and intrusion pressure presented in Fig. $9 \mathrm{~g}$ and $\mathrm{h}$ show that after pre-soaked in oil for 5 days the HPCPU-coated mesh still exhibits very high separation efficiency and the same intrusion pressure. The strong endurance of the HPCPU-coated mesh provides the possibility of using the mesh for water/oil separation in industrial scale.

\section{Conclusions}

We have successfully synthesized the superhydrophilic and underwater superoleophobic HPCPU-coated mesh, which exhibits excellent water/oil separation capacity. The HPCPUcoated mesh was tested to separate water from the various water/oil mixtures with excellent separation efficiency. Here, we want to emphasize the durability and the fouling resistance of HPCPU. When the mesh was pre-treated with the acidic, alkaline, and salt solutions, the separation efficiency was kept up to $99.7 \%$. When the mesh was treated with hot water and presoaked in various oils, the separation efficiency maintained on the same level. With such property, the HPCPU-coated mesh could be used in very harsh environments, and real application in industrial scale.

\section{Conflicts of interest}

There are no conflicts to declare.

\section{Acknowledgements}

We thank the Natural Science Foundation of Xinjiang Uyghur Autonomous Region (2016D01C037).

\section{References}

1 B. M. Jenssen, Environ. Pollut., 1994, 86, 207-215. 
2 M. Cheryan and N. Rajagopalan, J. Membr. Sci., 1998, 151, 13-28.

3 M. A. Shannon, P. W. Bohn, M. Elimelech, J. G. Georgiadis, B. J. Marinas and A. M. Mayes, Nature, 2008, 452, 301-310.

4 Q. Ma, H. Cheng, A. G. Fane, R. Wang and H. Zhang, Small, 2016, 12, 2186-2202.

5 Z. Chu, Y. Feng and S. Seeger, Angew. Chem., Int. Ed., 2015, 54, 2328-2338.

6 X. Gao, L.-P. Xu, Z. Xue, L. Feng, J. Peng, Y. Wen, S. Wang and X. Zhang, Adv. Mater., 2014, 26, 1771-1775.

7 R. Boopathy, S. Shields and S. Nunna, Appl. Biochem. Biotechnol., 2012, 167, 1560-1568.

8 V. Broje and A. A. Keller, Environ. Sci. Technol., 2006, 40, 7914-7918.

9 M. O. Adebajo, R. L. Frost, J. T. Kloprogge, O. Carmody and S. Kokot, J. Porous Mater., 2003, 10, 159-170.

10 J. Chen, H. You, L. Xu, T. Li, X. Jiang and C. M. Li, J. Colloid Interface Sci., 2017, 506, 659-668.

11 F. Beshkar, H. Khojasteh and M. Salavati-Niasari, J. Colloid Interface Sci., 2017, 497, 57-65.

12 L. Feng, Z. Y. Zhang, Z. H. Mai, Y. M. Ma, B. Q. Liu, L. Jiang and D. B. Zhu, Angew. Chem., Int. Ed., 2004, 43, 2012-2014.

13 Z. J. Cheng, H. W. Liu, H. Lai, Y. Du, K. W. Fu, C. Li, J. X. Yu, N. Q. Zhang and K. N. Sun, ACS Appl. Mater. Interfaces, 2015, 7, 20410-20417.

14 F. J. Wang, S. Lei, C. Q. Li, J. F. Ou, M. S. Xue and W. Li, Ind. Eng. Chem. Res., 2014, 53, 7141-7148.

15 X. Zhu, Z. Zhang, B. Ge, X. Men, X. Zhou and Q. Xue, J. Colloid Interface Sci., 2014, 432, 105-108.

16 L. Zhang, L. Li and Z.-M. Dang, J. Colloid Interface Sci., 2016, 463, 266-271.

17 S. Yang, L. Chen, C. Wang, M. Rana and P.-C. Ma, J. Colloid Interface Sci., 2017, 508, 254-262.

18 B. Shang, Y. Wang, B. Peng and Z. Deng, J. Colloid Interface Sci., 2016, 482, 240-251.

19 D. Li and Z. Guo, J. Colloid Interface Sci., 2017, 503, 124-130.

20 H. Zhu, L. Gao, X. Yu, C. Liang and Y. Zhang, Appl. Surf. Sci., 2017, 407, 145-155.

21 W. Zhou, G. Li, L. Wang, Z. Chen and Y. Lin, Appl. Surf. Sci., 2017, 413, 140-148.

22 J. Yang, H. J. Song, X. H. Yan, H. Tang and C. S. Li, Cellulose, 2014, 21, 1851-1857.

23 J. Yang, Z. Z. Zhang, X. H. Xu, X. T. Zhu, X. H. Men and X. Y. Zhou, J. Mater. Chem., 2012, 22, 2834-2837.

24 J. Yang, L. T. Yin, H. Tang, H. J. Song, X. N. Gao, K. Liang and C. S. Li, Chem. Eng. J., 2015, 268, 245-250.

25 N. Valipour Motlagh, F. C. Birjandi, J. Sargolzaei and N. Shahtahmassebi, Appl. Surf. Sci., 2013, 283, 636-647.

26 H. Yu, Z. Lian, J. Xu, Y. Wan, Z. Wang, Y. Li, Z. Yu and Z. Weng, Appl. Surf. Sci., 2017, 437, 400-409.

27 Q. Wang, Y. Fu, X. Yan, Y. Chang, L. Ren and J. Zhou, Appl. Surf. Sci., 2017, 412, 10-18.

28 K. Hou, Y. Zeng, C. Zhou, J. Chen, X. Wen, S. Xu, J. Cheng, Y. Lin and P. Pi, Appl. Surf. Sci., 2017, 416, 344-352.

29 J. Li, R. Kang, X. Tang, H. She, Y. Yang and F. Zha, Nanoscale, 2016, 8, 7638-7645.
30 J. Li, L. Yan, H. Li, W. Li, F. Zha and Z. Lei, J. Mater. Chem. A, 2015, 3, 14696-14702.

31 J. Li, D. Li, Y. Yang, J. Li, F. Zha and Z. Lei, Green Chem., 2016, 18, 541-549.

32 J. Li, C. Xu, C. Guo, H. Tian, F. Zha and L. Guo, J. Mater. Chem. A, 2018, 6, 223-230.

33 Z. Xu, Y. Zhao, H. Wang, H. Zhou, C. Qin, X. Wang and T. Lin, ACS Appl. Mater. Interfaces, 2016, 8, 5661-5667.

34 X. Zheng, Z. Guo, D. Tian, X. Zhang, W. Li and L. Jiang, ACS Appl. Mater. Interfaces, 2015, 7, 4336-4343.

35 X. Zhou, Z. Zhang, X. Xu, F. Guo, X. Zhu, X. Men and B. Ge, ACS Appl. Mater. Interfaces, 2013, 5, 7208-7214.

36 Z.-Y. Luo, K.-X. Chen, J.-H. Wang, D.-C. Mo and S.-S. Lyu, J. Mater. Chem. A, 2016, 4, 10566-10574.

37 F. Chen, J. L. Song, Z. Liu, J. Y. Liu, H. X. Zheng, S. Huang, J. Sun, W. J. Xu and X. Liu, ACS Sustainable Chem. Eng., 2016, 4, 6828-6837.

38 H. L. Peng, J. N. Wu, Y. X. Wang, H. Wang, Z. Y. Liu, Y. L. Shi and X. H. Guo, Appl. Phys. A: Mater. Sci. Process., 2016, 122, 516.

39 Z. Xu, Y. Zhao, H. Wang, X. Wang and T. Lin, Angew. Chem., Int. Ed., 2015, 54, 4527-4530.

40 X. Chen, J. A. Weibel and S. V. Garimella, Ind. Eng. Chem. Res., 2016, 55, 3596-3602.

41 L. Li, L. Liu, J. Lei, J. He, N. Li and F. Pan, J. Mater. Chem. A, 2016, 4, 12334-12340.

42 Y. Cao, N. Liu, W. Zhang, L. Feng and Y. Wei, ACS Appl. Mater. Interfaces, 2016, 8, 3333-3339.

43 Z. X. Xue, S. T. Wang, L. Lin, L. Chen, M. J. Liu, L. Feng and L. Jiang, Adv. Mater., 2011, 23, 4270-4273.

44 Y. Q. Liu, Y. L. Zhang, X. Y. Fu and H. B. Sun, ACS Appl. Mater. Interfaces, 2015, 7, 20930-20936.

45 Y. Dong, J. Li, L. Shi, X. B. Wang, Z. G. Guo and W. M. Liu, Chem. Commun., 2014, 50, 5586-5589.

46 Z.-Q. Dong, B.-J. Wang, M. Liu, X.-h. Ma and Z.-L. Xu, RSC $A d v .$, 2016, 6, 65171-65178.

47 J. Li, L. Yan, W. Li, J. Li, F. Zha and Z. Lei, Mater. Lett., 2015, 153, 62-65.

48 S. Zhang, F. Lu, L. Tao, N. Liu, C. Gao, L. Feng and Y. Wei, ACS Appl. Mater. Interfaces, 2013, 5, 11971-11976.

49 Z. Cheng, H. Lai, Y. Du, K. Fu, R. Hou, C. Li, N. Zhang and K. Sun, ACS Appl. Mater. Interfaces, 2014, 6, 636-641.

50 N. Liu, Y. Cao, X. Lin, Y. Chen, L. Feng and Y. Wei, ACS Appl. Mater. Interfaces, 2014, 6, 12821-12826.

51 F. Zhang, W. B. Zhang, Z. Shi, D. Wang, J. Jin and L. Jiang, Adv. Mater., 2013, 25, 4192-4198.

52 S. Yanlong, Y. Wu, F. Xiaojuan, W. Yongsheng, Y. Guoren and J. Shuping, Appl. Surf. Sci., 2016, 367, 493-499.

53 Y. Zhang, W. Li, R. Wu and W. Wang, RSC Adv., 2017, 7, 33701-33707.

54 W. Li, X. Jiang, R. Wu and W. Wang, Polym. J., 2017, 49, 263271.

55 Y. Zhang, X. Jiang, R. Wu and W. Wang, J. Appl. Polym. Sci., 2016, 133, 43534.

56 Z. Wang, W. Li, X. Yang, J. Cao, Y. Tu, R. Wu and W. Wang, Mater. Today Commun., 2018, 17, 246-251. 